\title{
Morphological Evolution of Calcium Phosphate Crystals with the Assistance of Motif-Programmed Artificial Proteins
}

\author{
Toru Tsuji ${ }^{1} *$ and Kiyotaka Shiba ${ }^{1}$ \\ ${ }^{1}$ Division of Protein Engineering, Cancer Institute, Japanese Foundation for Cancer Research, Koto, Tokyo 135-8550, \\ Japan. torutsuj@educ.cc.keio.ac.jp; kshiba@jfcr.or.jp
}

Hydroxyapatite (HA) is a major component of bone and teeth. It has been proposed that octacalcium phosphate (OCP) and/or amorphous calcium phosphate are precursors of HA, and its evolution is regulated by specific proteins. Understanding the mechanisms by which biomolecules regulate the mineralization of calcium phosphates will provide guiding principles for fabricating biomaterials with properties similar to

those of biological apatite. Dentin matrix protein 1 (DMP1) is the protein involved in bone and dentin mineralization. Synthetic peptides analogous to DMP1 motifs A and B have been shown to accelerate HA formation when they were immobilized on a glass surface. We have previously constructed artificial proteins by combinatorially assembling these two motifs, and found that one of the created proteins, namely, \#64, accelerated HAP and OCP formation without immobilization. In this report, we investigated the effects of \#64 derivatives on the crystal morphologies of calcium phosphates in mineralization experiments. Four \#64 mutants, containing motifs in which the acidic residues were substituted with neutral residues, showed different levels of mineralization activity, and the resultant calcium phosphate precipitates had distinct morphologies, indicating a possible evolutionary path of calcium phosphates precipitated in the presence of motif-programmed artificial proteins.

Key words: calcium phosphate, biomaterials, crystal growth, protein engineering, motif programming

\section{INTRODUCTION}

Hydroxyapatite $\left[\mathrm{Ca}_{10}\left(\mathrm{PO}_{4}\right)_{6}(\mathrm{OH})_{2}, \mathrm{HA}\right]$ is a major component of bone and teeth. It has been proposed that $\mathrm{HA}$ evolves from octacalcium phosphate $\left[\mathrm{Ca}_{8}\left(\mathrm{HPO}_{4}\right)_{2}\left(\mathrm{PO}_{4}\right)_{4} \cdot 5 \mathrm{H}_{2} \mathrm{O}\right.$, OCP $]$ and/or amorphous calcium phosphate (ACP) by transformation ${ }^{1}$, and this process is robustly regulated by specific proteins called biomineralization proteins $s^{2}$. An understanding of the mechanisms by which these proteins regulate the mineralization of calcium phosphates will provide guiding principles for fabricating biomaterials with the properties similar to those of biological apatite.

Dentin matrix protein 1 (DMP1) is present in the extracellular matrices of bone and dentin ${ }^{2}$. DMP1 as well as two synthetic peptides analogous to the two DMP1 motifs, namely, motif-A (ESQES) and motif-B (QESQSEQDS), have been shown to accelerate HA formation when they were immobilized on a glass substrate $^{3}$. However, they did not accelerate mineralization without immobilization ${ }^{4,5}$.

We have recently constructed a library of artificial proteins by combining motif-A and motif-B of DMP1 using our motif programming technique (Fig. 1a) ${ }^{5,6}$. One of the resultant proteins, namely, \#64, containing one motif-A and three motif-B sequences (Fig. 1b), had sufficient activity to accelerate HA or OCP formation without immobilization ${ }^{5}$.

To confirm the involvement of the motifs in the observed mineralization activity of \#64, we constructed four \#64 mutants in which the acidic residues [aspartic acid (D) and glutamic acid (E)] in one of the four motifs were substituted by neutral residues [aspargine $(\mathrm{N})$ and

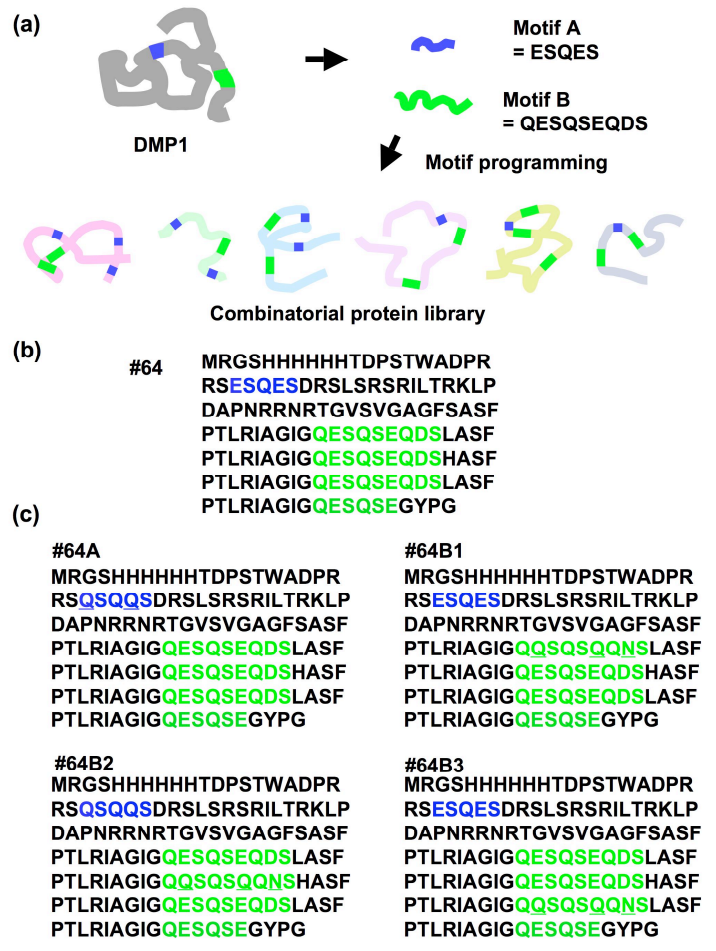

Fig. 1. Schematic representation of the motif programming technique for the construction of the combinatorial protein library. Two motifs from DMP1 were used for motif programming (a). Primary structures of \#64 (b) and its mutants (c). Substituted residues are indicated by underlined letters. 
glutamine (Q)] (Fig. 1c). Analyses of these four derivatives revealed that these four mutants exhibited distinct mineralization activities, but they are rather moderate compared to that of parental $\# 64^{5}$. Interestingly, these proteins induced the formation of morphologically distinct precipitates from calcium phosphate solutions. In this report, we observed the morphologies of calcium phosphate precipitates in the presence of these proteins under several conditions, and determined a possible evolutionary path of calcium phosphates in biomineralization.

\section{RESULTS and DISCUSSION}

We have performed precipitation experiments using calcium phosphate solutions with different degrees of supersaturation. All the mutants accelerated precipitation similarly, and no clear difference was observed among the mutants when we used a solution with a higher degree of supersaturation $\left(\left[\mathrm{Ca}^{2+}\right]=2.0 \mathrm{mM},\left[\mathrm{PO}_{4}^{3-}\right]=4.5 \mathrm{mM}\right.$, $\mathrm{pH}$ 8.0). Therefore, this study focused on experiments using a solution with a low degree of supersaturation $\left(\left[\mathrm{Ca}^{2+}\right]=1.8 \mathrm{mM},\left[\mathrm{PO}_{4}{ }^{3-}\right]=1.1 \mathrm{mM}, \mathrm{pH} 7.4\right)$. The precipitation reaction was initiated by mixing equal volumes of calcium and phosphate solutions (500 $\mu$ l each). Different amounts of the proteins were dissolved in the phosphate solutions before mixing with the calcium solutions. The solutions were incubated at a constant temperature $\left(25^{\circ} \mathrm{C}\right)$ without agitation during the reaction. After the reaction, samples were collected, and examined by scanning electron microscope (SEM).

We confirmed that calcium phosphates did not precipitate from the solution even after 10 days of incubation in the absence of the proteins. However, spherical calcium phosphate aggregates with a diameter of $\sim 20 \mu \mathrm{m}$ precipitated in the presence of $1.6,8.0$ and 40 $\mu \mathrm{g} / \mathrm{ml}$ of \#64 protein within 5 days of incubation (Figs. $2 \mathrm{a}$ and $\mathrm{b})$. The aggregates appeared as blade-shaped crystals, and were identified as octacalcium phosphate (OCP), a precursor of hydroxyapatite, by X-ray diffraction (XRD) analysis ${ }^{5}$. Along with the precipitation, the $\mathrm{pH}$ values of the solutions decreased from 7.4 to 6.7. Because OCP is stabilized kinetically in slightly acidic solutions $^{7}$, formation of OCP under our experimental conditions is consistent with a general notion. Calcium phosphate did not precipitate in the presence of a higher concentration of \#64 $(200 \mu \mathrm{g} / \mathrm{ml})$. Similar results have been reported, where amelogenin, a protein involved in enamel formation, accelerated OCP formation, whereas this protein did not show any activity at concentrations higher than $130 \mu \mathrm{g} / \mathrm{ml}^{8}$. It has been proposed that higher amounts of protein sequester calcium and/or phosphate ions, and this lowers the actual ionic concentrations, resulting in decreased degree of supersaturation of the solution $^{8}$. DMP1, synthetic peptides of motif-A and motifs- $\mathrm{B}$, and commercially available proteins such as bovine serum albumin (BSA), lysozyme, and phosvitin did not exhibit the mineralization activity under the same conditions 5 .

The results of the precipitation experiments performed in the presence of \#64 mutants are summarized in Fig. 3a. All the mutants accelerated precipitation. However, higher protein concentrations of

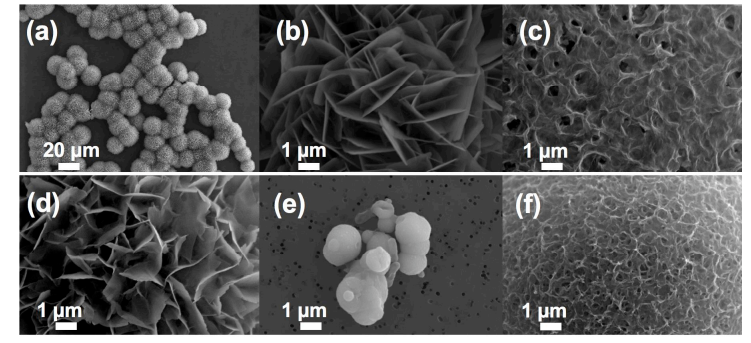

Fig. 2. SEM observation of calcium phosphates precipitated in the presence of artificial proteins. Spherical aggregates of blade-shaped OCP formed in the presence of $40 \mu \mathrm{g} / \mathrm{ml}$ of \#64 during a 5 day incubation (a, b). Surface of the spherical aggregate formed in the presence of $200 \mu \mathrm{g} / \mathrm{ml}$ of \#64B1 after 5 (c) and 10 (d) days of incubation. Globular particles formed in the presence of $1 \mathrm{mg} / \mathrm{ml}$ of \#64B2 after 6 days of incubation (e). Surface of a spherical aggregate formed in the presence of $1 \mathrm{mg} / \mathrm{ml}$ of \#64B2 after 11 days of incubation (f).

(a)

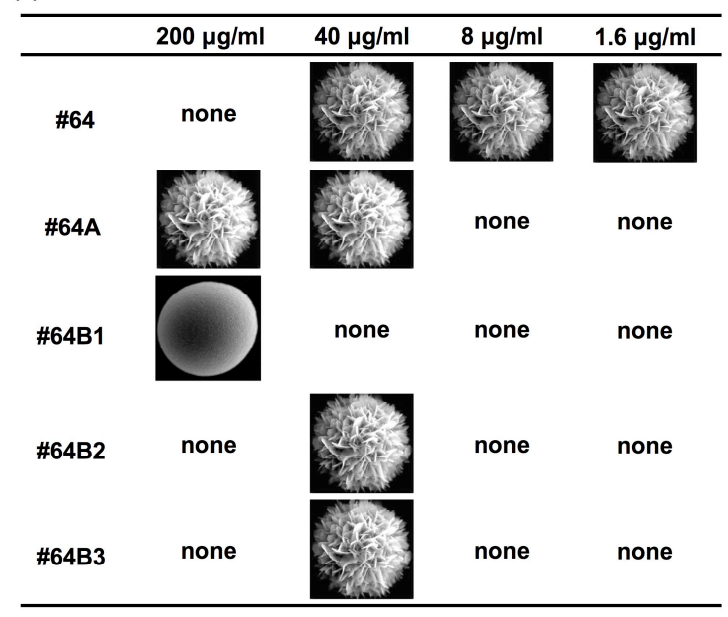

(b)

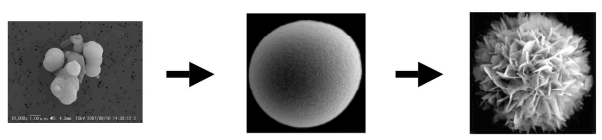

Fig. 3. Morphologies of calcium phosphates precipitated in the presence of artificial proteins. Summary of the precipitation experiments in the presence of indicated concentrations of artificial proteins during a 5 day incubation. The blade type morphology is represented by a single photograph (a). Deduced morphological evolution of calcium phosphates in the presence of artificial proteins (b).

the mutants were required compared to the parental \#64 concentration. Amino acid substitutions within the DMP1 motifs thus decreased the mineralization activity of \#64, indicating that the motifs play a crucial role in the mineralization activity of \#64. Three mutants, \#64A, \#64B2, and \#64B3, showed mineralization activity at protein concentration of $40 \mu \mathrm{g} / \mathrm{ml}$, whereas, \#64B1 exhibited activity only when $200 \mu \mathrm{g} / \mathrm{ml}$ of the protein was present in the solution. Activity of \#64B1 was the weakest among the mutants. These results suggested that 
among the embedded motifs in \#64, the contribution of motif-B1 to the mineralization activity was the most significant. It is noteworthy that the three motif-B sequences in \#64 did not contribute equally to the mineralization activity. This may be due to the effect of local environments around the motifs, which are probably different in the case of each motif. Similar "context effect" has often been observed in motif-programmed experiments ${ }^{9}$

Interestingly, the morphology of spherical aggregates obtained in the presence of \#64B1 was different from those of other precipitates. The aggregates had wavy surfaces and not blade-shaped ones (Figs. 2c and 3a). Because, the mineralization activity of $\# 64 \mathrm{~B} 1$ is weaker than that of other proteins, the precipitates are probably intermediates in the formation of the spherical OCP aggregates. We therefore continued the reaction, and the morphology of the precipitates was observed again after 5 days of incubation. During the incubation period, wavy calcium phosphate precipitates evolved into blade-shaped OCP crystals (Fig. 2d). Thus spherical aggregates with wavy surfaces are precursors of blade-shaped OCP aggregates.

However, Gungormus et al have proposed that aggregates of globular particles with $\mu \mathrm{m}$ size were precursors of the spherical aggregates of $\mathrm{OCP}^{10}$. To address this discrepancy, we performed additional experiments, in which precipitates were formed in the presence of $1 \mathrm{mg} / \mathrm{ml}$ of \#64B2. The presence of high amounts of the protein prevented the formation of spherical OCP aggregates during the 6 days of incubation. However, aggregates of globular particles whose size and morphology were very similar to the one reported by Gungormus et al were obtained (Fig. 2e). Furthermore, we have confirmed that the globular particles were transformed into spherical aggregates with wavy surfaces after further 5 days of incubation (Fig. 2f). Thus, the particles observed by Gungormus et al could be precursors of the spherical aggregates with wavy surfaces. On the basis of these observations, we propose the morphological evolution of calcium phosphates assisted by artificial proteins as shown in Figure 3b.

Although proteins used in this study had different levels of acceleration activity for precipitation, final crystals obtained in the presence of the proteins were similar to each other. Thus substitutions of amino acids in DMP1 motifs of \#64 affected only velocity of crystal formation, and not properties of final crystals. These results suggest that DMP1 motifs have activity to mediate only early stages of crystal formation, which is consistent with the notion revealed by our previous study, where \#64 was found to change the mechanism of nucleation of crystalline calcium phosphate ${ }^{5}$.

In summary, we investigated the morphologies of calcium phosphates precipitated in the presence of mineralization proteins under various conditions. The proteins had different levers of mineralization activity, and calcium phosphates with different morphologies were obtained depending on the protein species, concentration, and reaction time. On the basis of these observations, we propose the morphological evolution of calcium phosphates precipitated in the presence of artificial proteins. Calcium phosphates initially precipitated as aggregates of globular particles with $\mu \mathrm{m}$ size. The initial precipitates evolved into spherical aggregates with wavy surfaces with diameters of several tens of $\mu \mathrm{m}$. Finally, blade-shaped OCP formed on the surfaces of the spherical aggregates.

\section{MATERIALS AND METHODS}

3.1 Purification of artificial proteins.

Motif-programmed proteins (tagged with hexahistidine) were expressed in E.coli, and purified under denaturing conditions using cobalt affinity chromatography as described previously ${ }^{5}$. The proteins were dialyzed against $0.001 \mathrm{~N} \mathrm{HCl}$ and freeze-dried. The purity and molecular mass of the proteins were confirmed by electrophoresis and mass spectrometry.

3.2 Precipitation experiments

The precipitation reaction was initiated by mixing $\mathrm{KH}_{2} \mathrm{PO}_{4}$ solution and $\mathrm{CaCl}_{2}$ solution in a $2.0 \mathrm{ml}$ plastic tube at $25{ }^{\circ} \mathrm{C}$. The ionic concentrations are described in the main text. Before mixing the solutions a certain amount of protein was dissolved in $\mathrm{KH}_{2} \mathrm{PO}_{4}$ solution, and the $\mathrm{pH}$ of the solution was adjusted to $7.40 \pm 0.02$. The samples were incubated at $25^{\circ} \mathrm{C}$.

3.3 Characterization of calcium phosphate particles

Precipitates were collected by filtration, rinsed with milli-Q water, and air-dried at room temperature. Morphologies of the precipitates were analyzed by SEM (VE-8800; KEYENCE).

\section{REFERENCES}

[1] L. Wang and G. H. Nancollas, Chem. Rev., 108, 4628-69 (2008).

[2] A. George and A Veis, Chem. Rev., 108, 4670-93 (2008).

[3] G. He, T. Dahi, A. Veis and A. George, Nat. Mater. 2 , 552-8 (2003).

[4] G. He, S. Gajjeraman, D. Schultz, D. Cookson, C. Qin, W. T. Butler, J. Hao and A. George, Biochemistry, 44, 16140-8 (2005).

[5] T. Tsuji, K. Onuma, A. Yamamoto, M. Iijima, K. Shiba, Proc. Natl. Acad. Sci. USA, 105, 16866-70 (2008).

[6] K. Shiba, Y. Takahashi, T. Noda, Proc. Natl. Acad. Sci. USA, 94, 3805-10 (1997).

[7] M. Iijima and J. Moradian-Oldak, J. Mater. Chem., 14, 2189-99 (2004).

[8] B. J. Tarasevicha, C. J. Howard, J. L. Larson, M. L. Snead, J. P. Simmer, M. Paine and W. J. Shaw, J. Crystal Growth, 304, 407-15 (2007).

[9] H. Saito, T. Honma, T. Minamisawa, K. Yamazaki, T. Noda, T. Yamori, K. Shiba, Chem. Biol., 11, 765-73 (2004).

[10] M. Gungormus, H. Fong, W. Kim, J. S. Evans, C. Tamerler and M. Sarikaya, Biomacromolecules, 9, 966-73 (2008).

\section{ACKNOWLEDGMENTS}

This work was supported in part by Grant-in-Aid for Young Scientists (B) and (A) from the Japan Society for the Promotion of Science to T.T. (18710164, 21681026).

(Received January 7, 2009; Accepted February 15, 2010) 